\title{
Adjoint-Based Optimization of a Foam EOR Process
}

\author{
M. Namdar Zanganeh (Xodus Group), J.F.B.M. Kraaijevanger* (Shell Global \\ Solutions International), H.W. Buurman (Shell Global Solutions \\ International), J.D. Jansen (TU Delft) \& W.R. Rossen (TU Delft)
}

\section{SUMMARY}

We apply adjoint-based optimization to a Surfactant-Alternating-Gas foam process using a linear foam model introducing gradual changes in gas mobility and a nonlinear foam model giving abrupt changes in gas mobility as function of oil and water saturations and surfactant concentration.

For the linear foam model, the objective function is a relatively smooth function of the switching time. For the nonlinear foam model, the objective function exhibits many small-scale fluctuations. As a result, a gradient-based optimization routine could have difficulty finding the optimal switching time.

For the nonlinear foam model, extremely small time steps were required in the forward integration to converge to an accurate solution to the semi-discrete (discretized in space, continuous in time) problem. The semi-discrete solution still had strong oscillations in gridblock properties associated with the steep front moving through the reservoir. In addition, an extraordinarily tight tolerance was required in the backward integration to obtain accurate adjoints.

We believe the small-scale oscillations in the objective function result from the large oscillations in gridblock properties associated with the front moving through the reservoir. Other EOR processes, including surfactant EOR and near-miscible flooding, have similar sharp changes, and may present similar challenges to gradient-based optimization. 


\section{Introduction}

A hydrocarbon reservoir should be produced in an efficient, and, if possible, optimal manner. Our objective here is maximizing the cumulative oil production in a foam surfactant-alternating-gas (SAG) process (Shan and Rossen 2004) by finding the optimum switching time between the surfactant and gas injection cycles. Foam is a means of improving sweep efficiency that reduces the gas mobility by capturing gas in foam bubbles and hindering its movement (Rossen 1996). Specifically, we investigate the performance of adjoint-based optimization applied to this enhancedoil-recovery (EOR) process. This work is the first attempt to apply optimal control theory to foam EOR.

Adjoint-based schemes are very efficient for gradient calculations and are particularly useful in optimization problems where a large number of controllable parameters must be optimized. Here we use the Shell in-house reservoir simulator MoReS (hereafter referred to as the simulator). The adjoint routine in the simulator has been extensively validated for waterflooding (Kraaijevanger et al. 2007; Van Essen et al. 2010) and also recently for polymer flooding (Van Doren et al. 2011). In principle, adjoint-based optimization could be applied to many other EOR processes.

In Section 2 of this paper, we first review briefly the history of application of optimal control theory in petroleum engineering and EOR in particular. Next, in Section 3, we define the problem and associated simulation models. Section 4 describes and explains oscillations that we observed in the numerical simulation of these models. The effect of the switching time on cumulative oil production is investigated in Section 5. In particular, we discuss the application of the adjoint method for calculating the gradient of the cumulative oil production with respect to switching time. In Section 6, we investigate the performance of a gradient-based optimization routine in finding the switching time that maximizes the cumulative oil production. We examine the performance in a number of cases varying in the simulation mode (i.e., 1D or 3D) and the foam model (i.e., linear or nonlinear). We end the paper with conclusions and implications in Section 7.

\section{Optimal Control Theory}

Optimal control theory is a mathematical discipline aiming at optimal control of time-dependent systems governed by partial or ordinary differential equations. In optimal control theory it is common practice to use gradient-based optimization methods, where the gradient is obtained with adjoint techniques involving the solution of adjoint equations backward in time. Originally developed for nonlinear trajectory optimization in aerospace engineering, optimal control theory emerged in petroleum engineering during the 1970s for computer-assisted history matching; see Oliver et al. (2008) for an overview. Starting about a decade later the methodology was used for the optimization of tertiary recovery processes such as surfactant, polymer, $\mathrm{CO}_{2}$ or steam flooding; see e.g. Ramirez et al. (1984), Fathi and Ramirez (1984, 1987), Ramirez (1987), Mehos and Ramirez (1989), Liu et al. (1993), and Liu and Ramirez (1994). Applications to waterflood optimization followed soon and are continuing today (see Jansen (2011) for a recent overview), but EOR applications seem to have come to a standstill.

A possible explanation is that applying optimal control theory can lead to complications. A certain method may work well for simple examples, but result in suboptimal solutions in more-complex systems. A complicating factor in the comparison of earlier papers is that there are three different approaches to derive the adjoint equations: 1) Starting from the governing partial differential equations (PDEs) for the time-dependent system, derive the adjoint PDEs whereafter the 'forward-intime' system PDEs and 'backward-in-time' adjoint PDEs are both discretized in space and time in order to solve them numerically. 2) Starting from the ordinary differential equations (ODEs) obtained by spatial discretization of the governing PDEs, derive the adjoint ODEs whereafter the 'forward' and adjoint ODEs are both discretized in time in order to solve them numerically. 3) Starting from the algebraic equations obtained by spatial and time discretization (either sequential or simultaneous) of the governing PDEs, derive the adjoint algebraic equations whereafter the 'forward' and adjoint algebraic equations are solved numerically. Depending on the details of the discretization methods for the forward and adjoint equations these approaches may lead to differences in numerical performance. Ramirez et al. (1984) and Fathi and Ramirez (1984) had difficulty in solving the adjoint equations, obtained using the first approach, in surfactant-flooding optimization. The adjoint PDEs had non- 
smooth coefficients caused by the physical nature of the governing forward PDEs, making it difficult to find a stable numerical adjoint scheme. Fathi and Ramirez associated this problem with the discontinuity in the adjoint equation coefficients caused by two shocks (a Buckley-Leverett shock and a shock at the surfactant front). In Fathi and Ramirez $(1984,1986)$ this problem was circumvented by replacing the adjoint equations with simpler, quasilinear approximations. Later, Fathi and Ramirez (1987) applied optimal control theory to micellar/polymer flooding using both the first and the third approach. Also here they reported problems with discontinuities in the coefficients of the adjoint PDEs. As a remedy they derived adjoint equations using the third approach. They provided some theoretical comparisons between the approaches but did not give details about their comparative computational performance.

For 2D steamflood optimization Liu et al. (1993) reported that finding a stable adjoint solution (using the third approach, but with different time steps sizes to solve the forward and the adjoint equations) could be a significant problem. We note that the use of different step sizes requires interpolation of the states, computed during the forward simulation, during the backward simulation. This gives it a flavour of the second approach. Liu and Ramirez (1994) extended the analysis of steamflood optimization to $3 \mathrm{D}$ and they found that a too-large time-step size for the adjoint solution can cause non-smooth optimal control strategies. They claimed that refinement of the time step should smooth the control strategies. They, moreover, argued that the adjoint solution may not necessarily result in correct gradients when computed with the same time-step sizes as used to compute the forward solution. This is contrary to the commonly held opinion that a correct adjoint solution should be based on exactly identical spatial and time discretization choices for the forward and backward equations.

Sudaryanto (1998) applied approach three in miscible EOR and faced problems in finding the optimum switching time (i.e. the optimal moment in time to terminate gas injection in one injector and start it in another injector) for a displacement with variable mobility. He stated that these problems are possibly caused by numerical errors in solving large systems of state and adjoint equations but did not analyse the problem in depth.

We also encountered problems related to coefficient values that are strongly nonlinear functions of the states, although of a somewhat different manifestation. The main issue was the magnitude of the relative tolerance for the adjoint linear solver, as discussed in Section 6 below. In addition, we encountered non-smooth adjoint gradients with various fluctuations, as described also in Section 6.

\section{Problem Statement}

We model a foam-displacement process in 1D and 3D. Specifically, we seek the period of injection of the surfactant slug in a single-cycle SAG flood that maximizes cumulative oil production. We represent the duration of the surfactant slug by the switching time $\left(t_{s}\right)$. Before time $t_{s}$ there is no gas or foam in the reservoir. At $t_{s}$, surfactant injection is terminated and gas injection starts. Foam forms in the reservoir where gas, water and surfactant are all present in sufficient amounts. The total period of injection is fixed; thus as $t_{s}$ increases, the period of liquid injection increases and that of gas injection decreases.

The 3D reservoir is box-shaped, $100 \mathrm{~m} \times 100 \mathrm{~m} \times 30 \mathrm{~m}$ (modelled with $10 \times 10 \times 10$ gridblocks), sealed on all bounding surfaces, with one injection and one production well, located at opposite corners of the reservoir. Both wells are vertical, located in the middle of the corner gridblocks, and are perforated in the entire interval. Both wells operate at a constant prescribed bottomhole pressure; thus foam affects not only sweep efficiency but also injection and production rates. In 1D simulations, the reservoir is $100 \mathrm{~m} \times 1 \mathrm{~m} \times 1 \mathrm{~m}(100 \times 1 \times 1$ gridblocks $)$. In the $1 \mathrm{D}$ simulations, wells are positioned in the middle of the first and last gridblocks. We use the Peaceman model for injectivity into and productivity from the well gridblocks. The reservoir is homogeneous in permeability and porosity.

We aim at investigating gas performance in sweeping oil. EOR is implemented usually after a period of waterflooding. Therefore, we set the initial oil saturation in the reservoir at the residual oil saturation to waterflood $\left(S_{\text {orw }}\right)$. As a result, oil is neither displaced nor produced before the switching time. Oil, water and gas are assumed to be completely immiscible. We assume that gas, though immiscible with oil, is capable of displacing oil; specifically, we set the residual oil saturation to gas flood $\left(S_{\text {org }}\right)$ lower than $S_{\text {orw }}$. We use the linear isoperm model (Baker 2008) for three-phase relative permeabilities. These and other simulation parameters are summarized in the Appendix. The gas 
phase is slightly compressible in the simulations (i.e., $c_{g}=1.68 \mathrm{E}-8 \mathrm{~Pa}^{-1}$ ). Water and oil are incompressible.

For the modelling of surfactant, the simulator represents it as a normal active component in the water phase. As will be discussed below, the surfactant concentration plays an important role in our foam model. We do not include adsorption of surfactant in our model. The level of surfactant adsorption affects the value of the optimal slug size but does not fundamentally alter the optimization process. Therefore for simplicity we exclude it here.

We use two foam models, one with abrupt changes (nonlinear model) and one without (linear model). In both cases, foam is not explicitly modelled as such, but only implicitly, by introducing a reduction factor of the gas mobility in our oil/water/gas system. In these foam models, the relative gas mobility in the absence of foam $\left(\lambda_{r g}^{0}\right)$ is rescaled to the relative gas mobility in the presence of foam $\left(\lambda_{r g}\right)$ by multiplying it by a dimensionless reduction factor (Eq. (A-2) in the Appendix); see also Rossen et al. 1999, Namdar Zanganeh et al. 2011. Our foam models incorporate the effects of water saturation, oil saturation, and surfactant concentration on the reduction in gas relative mobility as illustrated in Fig. 3.1. In the nonlinear foam model, foam is weakened at low water saturations according to Eq. (A-3). Note that foam strength equals zero at the connate water saturation according to this equation; otherwise the performance of the SAG flood can be greatly distorted (Namdar Zanganeh et al. 2011). In addition, foam is killed at high oil saturations as prescribed in Eq. (A-4). The foam sensitivity to surfactant concentration is defined by Eq. (A-5). In the nonlinear foam model, foam strength changes abruptly with small changes in water saturation, in agreement with laboratory data (Cheng et al. 2000). It also changes abruptly with changes in surfactant concentration, for reasons discussed in Namdar Zanganeh (2011). In fact, laboratory data suggest an even sharper change in foam strength with water saturation than modelled here (Cheng et al. 2000), but, as we will see, the nonlinear functions we use here are already challenging enough for the simulator. These types of functions can lead to drastic changes in gas mobility in individual gridblocks as fronts pass (Rossen et al. 1999; Namdar Zanganeh 2011). Note that all these functions are differentiable over the range of conditions that may be encountered in the simulation.

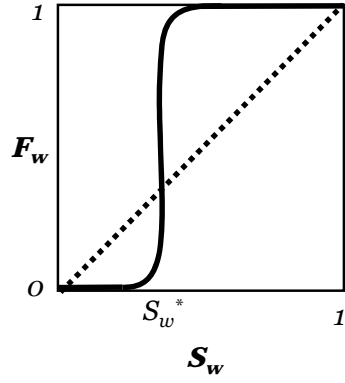

(a)

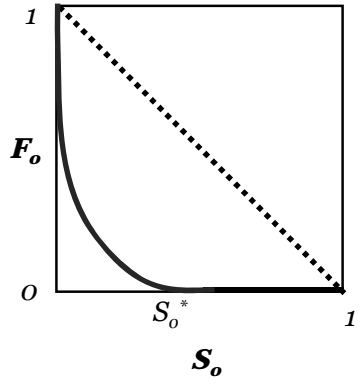

(b)

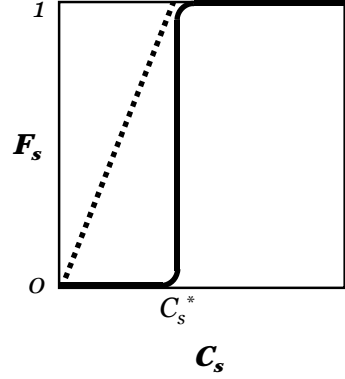

(c)

Figure 3.1 Foam models: The linear foam model is illustrated by dashed lines and the nonlinear foam model by solid lines. A value of $F_{i}=0$ means foam has no effect on gas mobility (Eq. (A-2)). (a) Foam sensitivity to water saturation, nonlinear model: foam is weakened at $S_{w} \leq S_{w}{ }^{*}=$ fmdry (Eq. (A-3)). (b) Foam sensitivity to oil saturation, nonlinear model: foam is killed at $S_{o} \geq S_{o}{ }^{*}=$ fmoil (Eq. (A-4)). (c) Foam sensitivity to surfactant concentration, nonlinear model: $F_{s}=1$ for $C_{s} \geq C_{s}^{*}$, where $C_{s}{ }^{*}=0.0012$ is half the injected concentration of 0.0024 (weight fraction) (Eq. (A-5)). The foam models are described further in the Appendix.

In general, optimization of an objective function can be carried out by combining an adjoint routine and an optimization routine for finding the optimum. The adjoint routine is employed to calculate the analytical gradient of the objective function with respect to various static and dynamic parameters. Gradients point in the direction of the maximum increase in the objective function and a gradientbased optimization routine utilizes the gradients to search for the optimum.

As mentioned above, our objective is finding the switching time $\left(t_{s, o p t}\right)$ that maximizes the objective function, $\mathcal{J}$, which is defined as the cumulative oil production at the end of the simulation (time $T)$ :

$$
\mathcal{J}=\int_{0}^{T} Q_{o}(t) \mathrm{d} t
$$


where $Q_{o}$ is the surface volume rate of the oil phase and the end time $T$ is fixed. We choose a simple case with only one control variable (i.e., the switching time between the surfactant and gas injection cycles) for this investigation. With only one control variable, the global trend of the objective function $\mathcal{J}$ (i.e., the cumulative oil production) can be easily constructed with a reasonable number of simulation runs. With the global trend of $\mathcal{J}$ known, $t_{s, o p t}$ can be easily identified. This way, we have a solid reference case against which to evaluate the performance of the adjoint based optimization methodology.

\section{Numerical Oscillations in the Forward Solution}

In this section we discuss the strong oscillations that we observed in the numerical simulation of the $1 \mathrm{D}$ and 3D models with the nonlinear foam model. In these simulations, the sudden change in the injection composition from $100 \%$ surfactant solution to $100 \%$ gas at the switching time produces a period of strong oscillations in the gas-injection rate $\left(Q_{g}\right)$ shortly after the switch. We start with the discussion of the oscillations in the 1D model. The reason why we focus on these oscillations is that they are the cause of certain problems (discussed in Section 6) that we encountered with gradientbased optimization.

We start with the 1D model with the nonlinear foam model. Immediately after the switching time, the oil and water saturations throughout the reservoir are equal to $S_{o}=S_{\text {orw }}=0.3$ and $S_{w}=1-S_{\text {orw }}=0.7$, and the surfactant concentration (almost) to $C_{s}=0.0024$ (weight fraction). Referring to the nonlinear foam model (see Fig. 3.1 and Appendix), these conditions are favorable for foam formation $\left[S_{w}>S_{w}{ }^{*}=\right.$ $0.316, S_{o}<S_{o}{ }^{*}=0.4$ and $\left.C_{s}>C_{s}{ }^{*}=0.0012\right]$. Hence, when gas starts being injected in the first gridblock $(n=1)$, its mobility is very low. Since the simulator uses an upwind scheme for the flux calculations between gridblocks (i.e., the phase flux between two adjacent gridblocks uses the phase mobility of the upstream gridblock), gas can flow at significant rate to the next gridblock $(n=2)$ only if the gas mobility in the first gridblock has become significant. When the water saturation in the first gridblock has dropped below the critical value $S_{w}{ }^{*}=0.316$, gas mobility strongly rises in that gridblock and allows gas to flow to gridblock 2, causing a jump in the injection rate $Q_{g}$ (see Fig. 4.2a) and all gridblock pressures (in particular those downstream of gridblock 1, see Fig. 4.2b). This behavior repeats itself in the remaining gridblocks until $S_{w}$ drops below $S_{w}{ }^{*}$ in the last gridblock and gas breaks through to the production well. Consequently, we observe as many spikes as the number of gridblocks in the gas-injection rate profile in 1D (Fig. 4.1). The first 5 spikes of the profile with 100 gridblocks in Fig. 4.1 have been magnified in Fig. 4.2. We have also plotted the saturation profiles in the first 5 gridblocks. The moment at which the $n^{\text {th }}$ spike in $Q_{g}$ occurs corresponds to the moment at which water saturation in the $n^{\text {th }}$ gridblock drops below $S_{w}{ }^{*}=0.316$.

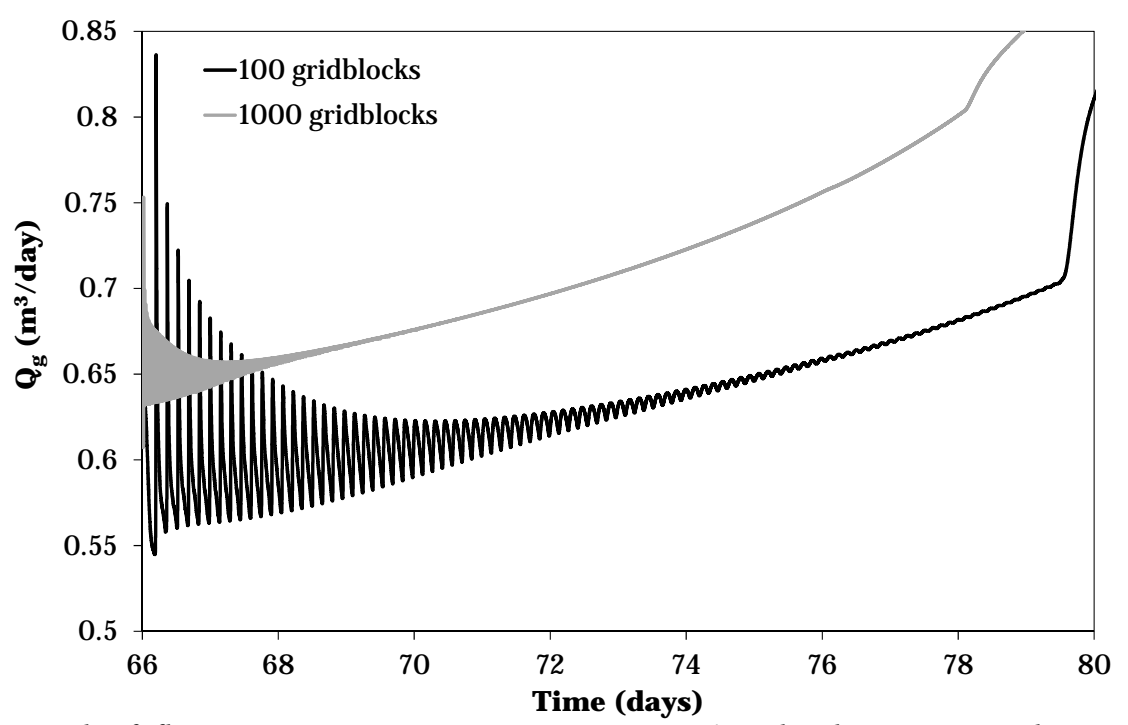

Figure 4.1 Period of fluctuating gas-injection rate in a $1 D$ displacement with $t_{s}=66$ days. The injection well operates at a constant prescribed bottomhole pressure. The number of spikes is equal to the number of gridblocks. This figure also illustrates the effect of grid size on the fluctuations. Finer grids reduce the amplitude of the spikes and shrink the period of large fluctuations to a period close to the switching time at $t_{s}=66$ days. 
We recall that we maintain a constant bottomhole pressure in the injection well ( $P_{w f}^{i n j}=250$ bar). Since $Q_{g}$ is proportional to the pressure difference between the wellbore and $P_{1}$, this pressure difference reflects the fluctuations in $P_{1}$. Although the fluctuations in $P_{1}$ are small and not noticeable in Fig. $4.2 \mathrm{~b}$, once those small pressure differences are multiplied by the large total mobility in the well gridblock, the product produces noticeable fluctuations in the gas-injection rate.

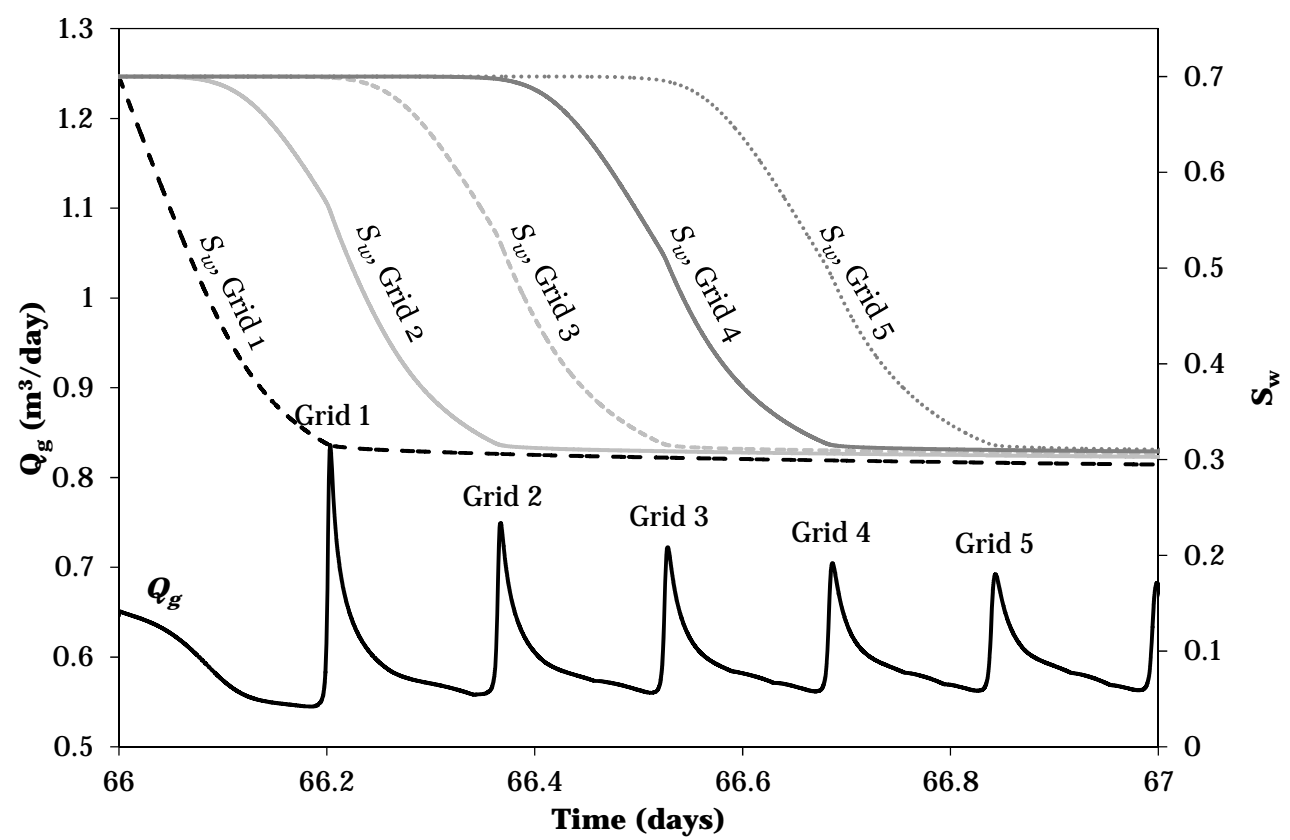

(a)

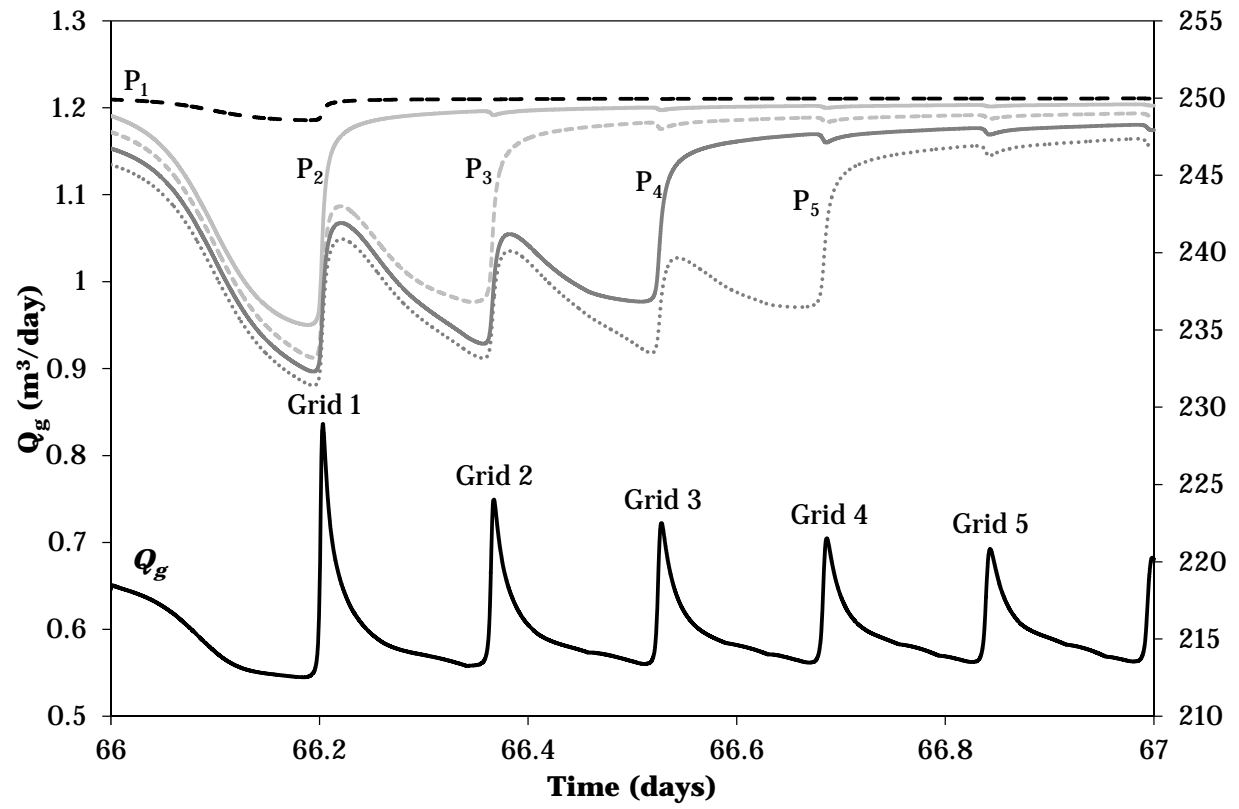

(b)

Figure 4.2 Magnification of the first 5 spikes in gas-injection rate in Fig. 4.1 (with 100 gridblocks): relation to water saturation (a) and pressure (b) in each gridblock. The moment at which the $n^{\text {th }}$ spike in $Q_{g}$ occurs is the moment at which $S_{w}$ in the $n^{\text {th }}$ gridblock drops below $S_{w}{ }^{*}=0.316$.

In the remainder of this section we turn our attention to the 3D model, which has similar oscillations if we use the nonlinear foam model. Fig. 4.3 illustrates the $Q_{g}$ profile for a 3D reservoir in this case and the fluctuations caused by the presence of foam; in this example $t_{s}$ is 66 days. 


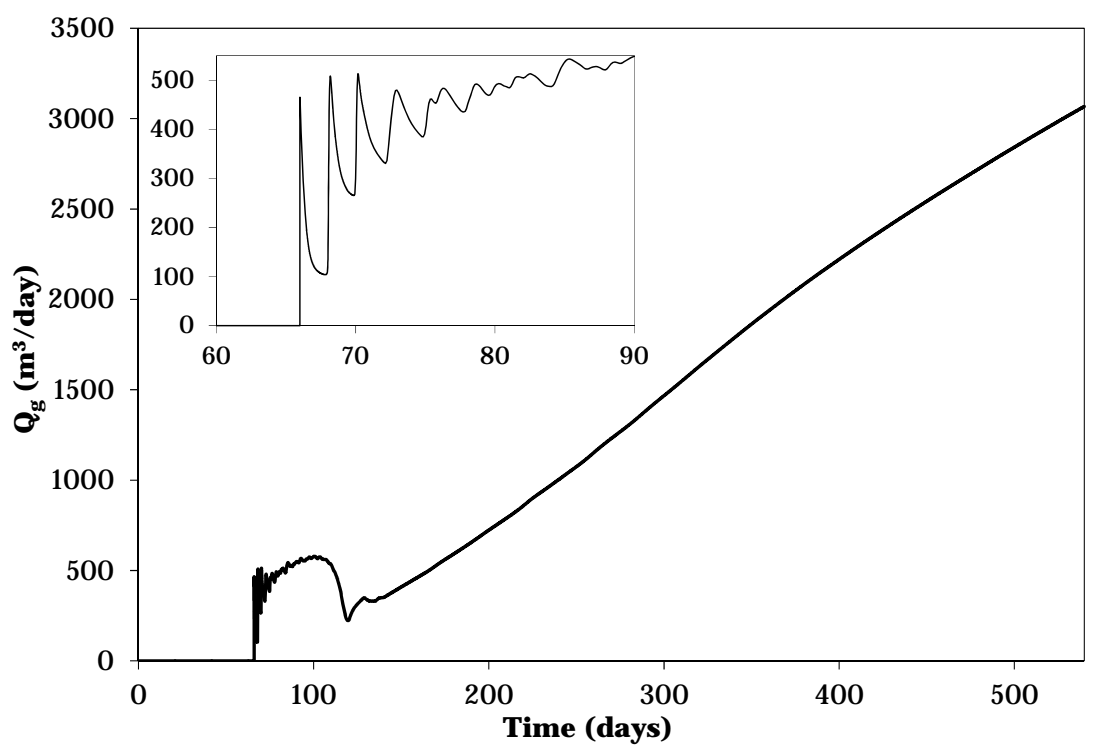

Figure 4.3 Gas-injection-rate profile versus time for a $3 D$ displacement. Simulation ends after 540 days and $t_{s}=66$ days. The inset magnifies the fluctuations right after the switching time. The reservoir consists of $1000(10 \times 10 \times 10)$ gridblocks.

In $3 \mathrm{D}$, relating the spikes to the gridblocks is less straightforward than in $1 \mathrm{D}$ because there are many more flow paths between injection and production well. As in the 1D model, grid refinement causes the number of spikes to grow while reducing the amplitude of the spikes and shrinking the period of large fluctuations to a period close to the switching time (see Fig. 4.4).

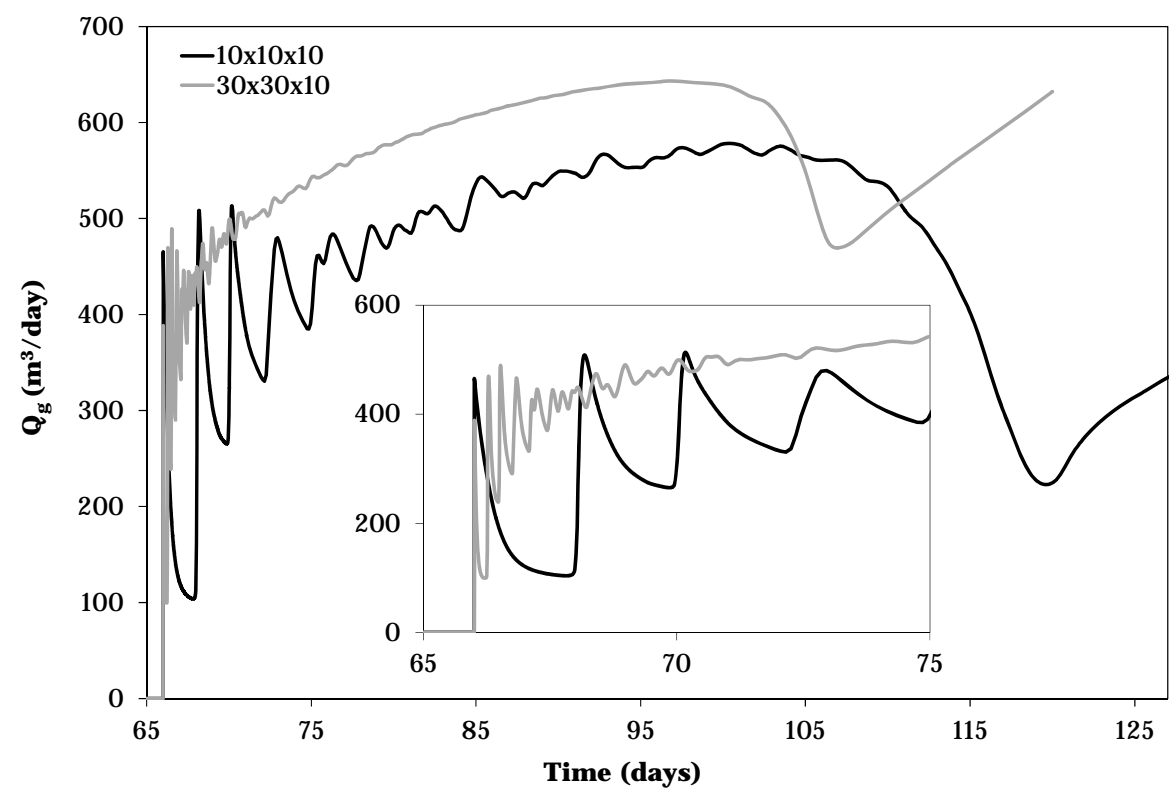

Figure 4.4: Effect of grid size on the fluctuations in the gas-injection profile in a 3D displacement shown in Fig. 4.3. Finer grids reduce the amplitude of the spikes and shrink the fluctuation interval to a region close to the switching time at $t_{s}=66$ days. In the refined case, each gridblock is 9 times smaller than the original gridblock.

We conclude this section with two remarks that apply to both 1D and 3D.

First, if a constant $Q_{g}$ is prescribed instead of a constant $P_{w f}^{i n j}$, fluctuations occur in the $P_{w f}^{i n j}$ profile to accommodate the prescribed $Q_{g}$ (cf. Rossen et al. 1999).

Secondly, the fluctuations do not vanish if we take (much) smaller time steps, but converge to the shapes as presented above in the limit $\Delta t \rightarrow 0$. Hence, these fluctuations are not related to the timestep size, but are due to the spatial discretization scheme used in the simulator. 


\section{Calculation of the Switching Time Gradient}

In this paper, the goal is finding the switching time $\left(t_{s, o p t}\right)$ that maximizes the cumulative oil production $(\mathcal{J})$. For gradient-based optimization this means that we must be able to compute the derivative $\mathrm{d} \mathcal{J} / \mathrm{d} t_{s}$.

The dynamic system of interest is a producing reservoir and its wells, described by a set of coupled algebraic and differential equations. After discretization in space (based on gridblocks) and time (using a time stepping method) we arrive at a discrete dynamic system described by the following set of algebraic equations,

$\mathbf{g}_{k}\left(\mathbf{x}_{k-1}, \mathbf{x}_{k}, t_{k-1}, t_{k}\right)=0 \quad(k=1, \ldots ., N)$,

where $k$ is the time-step index and $N$ is the number of time steps. At every time step $k$, the state vector $\left(\mathbf{x}_{k}\right)$ is a column vector that contains the saturations, pressures, and compositions of all reservoir gridblocks and well discretization nodes. During the forward run (i.e., normal simulation run), $\mathbf{x}_{k}$ is successively calculated by solving Eq. (5.1), starting with the initial state vector $\mathbf{x}_{0}$ at $t_{0}=0$.

Before we turn our attention to the derivation of an expression for the desired gradient $\mathrm{d} J / \mathrm{d} t_{s}$, we first introduce the closely related gradients $\mathrm{d} J / \mathrm{d} t_{k}($ for $k=1,2, \ldots, N)$. The latter gradients represent the amount of change in the objective function when $t_{k}$ slightly varies, but other times do not (Fig. 5.1). In other words, all the times except $t_{k}$ are kept fixed for the computation of the time gradient with respect to $t_{k}$.

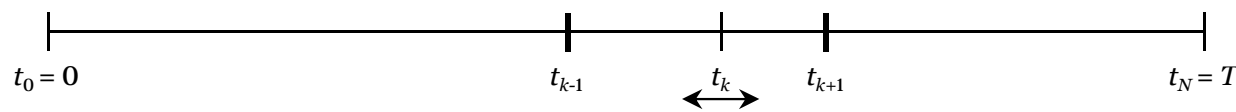

Figure 5.1 Schematic for calculation of the time gradient $\mathrm{d} J / \mathrm{d} t_{k}$ in the simulator. All the times except $t_{k}$ are kept fixed in the computation of the time gradient with respect to $t_{k}$.

Large values of $\mathrm{d} J / d t_{k}$ are expected only at the end time $(T)$ and at those times at which a change occurs in control settings like constraint type, constraint value, injection composition, etc. The value of $\mathrm{d} J / \mathrm{d} t_{k}$ for $k=N$ (i.e., at $t_{k}=t_{N}=T$ ) reflects the effect of changing the length of the simulation period.

The value $\mathrm{d} J / \mathrm{d} t_{k}$ at $t_{k}=t_{s}$ reflects the effect of prolonging or shortening the surfactant injection period on $\mathcal{J}$. If it is positive, prolonging the surfactant injection cycle leads to an increased $\mathcal{J}$. Conversely, if it is negative, it is not beneficial to extend the surfactant injection period. It seems reasonable to set the (desired) gradient $\mathrm{d} \mathcal{J} / \mathrm{d} t_{s}$ equal to $\mathrm{d} J / d t_{k}$, so that

$\frac{\mathrm{d} \mathcal{J}}{\mathrm{d} t_{s}}=\frac{\mathrm{d} \mathcal{J}}{\mathrm{d} t_{k}}$ (where $k$ is the index with $t_{k}=t_{s}$ ).

Note, however, that in actual simulations, a change in the switching time $t_{s}$ causes a change in many values $t_{k}$, depending on the chosen step-size strategy as discussed in Section 5.2. For a general stepsize strategy, all the values $t_{k}$ are dependent on the switching time $t_{s}$, leading to the following improved formula for $\mathrm{d} \mathcal{J} / \mathrm{d} t_{s}$,

$\frac{\mathrm{d} \mathcal{J}}{\mathrm{d} t_{s}}=\sum_{k=1}^{N}\left(\frac{\mathrm{d} \mathcal{J}}{\mathrm{d} t_{k}} \times \frac{\mathrm{d} t_{k}}{\mathrm{~d} t_{s}}\right)$

In practice, the difference of the estimates obtained with formulas (5.2) and (5.3) is usually negligible, because all values $\mathrm{d} \mathcal{J} / \mathrm{d} t_{k}$ (except those at $t_{k}=t_{s}$ and at $t_{k}=t_{N}=T$ ) are usually very small (as they represent time discretization errors), and our assumption of a fixed end time $t_{N}=T$ implies $\mathrm{d} t_{N} / \mathrm{d} t_{s}=0$. In certain cases, however, the difference can be significant. We will discuss this further in Section 5.2.

\subsection{Calculation of the Time Gradients $\mathrm{d} J / d t_{k}$ with the Adjoint Method}

For the calculation of the time gradients $\mathrm{d} J / \mathrm{d} t_{k}(\mathrm{k}=1,2, \ldots, \mathrm{N})$ we use the adjoint method (see e.g. Bryson and Ho 1975). Alternatively, the calculation of these gradients may also be done with the perturbation method or with the forward sensitivity method (see e.g. Kraaijevanger et al. 2007). In the forward sensitivity method and the adjoint method, the partial derivatives in Eqs. (5.4) to (5.7) below must be available in the source code of the simulator. 
The first step of the adjoint method is to solve the adjoint equations for the adjoint variables $\boldsymbol{\lambda}_{k}$ (see e.g. Bryson and Ho 1975 or Kraaijevanger et al. 2007),

$$
\begin{aligned}
& \lambda_{k} \mathbf{A}_{k}+\lambda_{k+1} \mathbf{B}_{k+1}=\frac{\partial \mathcal{J}}{\partial \mathbf{x}_{k}} \quad(k=1,2, \ldots, N-1), \\
& \lambda_{N} \mathbf{A}_{N}=\frac{\partial \mathcal{J}}{\partial \mathbf{x}_{N}},
\end{aligned}
$$

where the square matrices $\mathbf{A}_{k}$ and $\mathbf{B}_{k}$ are defined as

$$
\mathbf{A}_{k}=\frac{\partial \mathbf{g}_{k}}{\partial \mathbf{x}_{k}}, \quad \mathbf{B}_{k}=\frac{\partial \mathbf{g}_{k}}{\partial \mathbf{x}_{k-1}}
$$

The adjoint variables $\lambda_{k}$ are row vectors. The adjoint equations are solved backward in time, i.e., after solving (5.5) for $\lambda_{N}$, the equations in (5.4) are solved consecutively for $k=N-1, N-2, \ldots, 1$.

The adjoint equations are linear and solved by an iterative method. In the simulator, the convergence criterion is that the norm of the residual has become smaller than a user-specified relative tolerance times the norm of the initial residual.

Once the adjoint variables $\lambda_{k}$ are known, the gradients $\mathrm{d} J / \mathrm{d} t_{k}$ are computed as follows,

$$
\begin{array}{ll}
\frac{\mathrm{d} \mathcal{J}}{\mathrm{d} t_{k}}=\frac{\partial \mathcal{J}}{\partial t_{k}}-\lambda_{k} \frac{\partial \mathbf{g}_{k}}{\partial t_{k}}-\lambda_{k+1} \frac{\partial \mathbf{g}_{k+1}}{\partial t_{k}} & k=1,2, \ldots, N-1, \\
\frac{\mathrm{d} \mathcal{J}}{\mathrm{d} t_{N}}=\frac{\partial \mathcal{J}}{\partial t_{N}}-\lambda_{N} \frac{\partial \mathbf{g}_{N}}{\partial t_{N}} & k=N .
\end{array}
$$

\subsection{Dependence of $\mathrm{d} \mathcal{J} / \mathrm{d} t_{s}$ on the Chosen Step-Size Strategy}

The computation of the switching time gradient $\mathrm{d} J / d t_{s}$ is based on Eq. (5.3). In this section we illustrate this computation for a given step-size strategy.

We use the step-size strategy that starts with a given (small) initial step size $\Delta t^{\text {start }}$ and doubles the step size each time step until reaching a given maximum step size $\Delta t^{\max }$. We continue with this maximum step size until we reach the switching time (with a last time step possibly smaller than $\Delta t^{\max }$ ). At the switching time, we start again with the initial step size $\Delta t^{\text {start }}$, and repeat the same step-size strategy until we reach the simulation end time $T$. Fig. 5.2 shows an example with $t_{s}=4.7$ days and $T=10$ days.

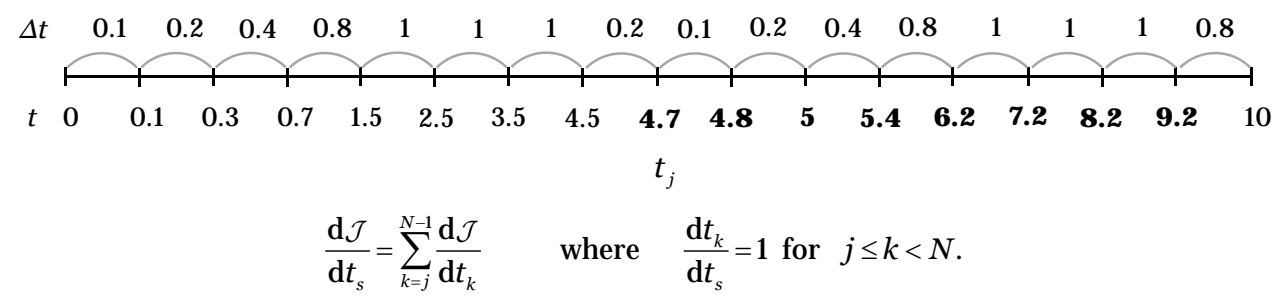

Figure 5.2 Simple example for calculating $\mathrm{d} J / d t_{s}$ where $t_{s}=4.7$ days and $T=10$ days. Small changes in the switching time affect only the time points $t_{k}$ in boldface. The term $\mathrm{d} t_{k} / \mathrm{d} t_{s}$ is equal to unity at these points. The time-step strategy is to start at both $t=0$ and $t=t_{s}$ with $\Delta t=\Delta t^{\text {start }}=0.1$ day and double it until reaching $\Delta t^{\max }=1$ day. Note that the steps are not of equal size.

If the sum of the intermediate gradients (i.e., the gradients corresponding to all $t_{k}$ with $t_{s}<t_{k}<T$ ) is large compared to the gradients at $t_{s}$ and $T$, it can be generally concluded that the time-discretization error is too large. In that case, a different step-size strategy should be considered. Unlike the gradients at $t_{s}$ and $T$, these intermediate gradients (and their sum) can be made arbitrarily small by choosing smaller time steps.

In our problem the time-step sizes in the vicinity of $t_{s}$ must be refined compared to the rest of the time grid. This causes a more accurate simulation in the hectic period right after the switch. The start of gas injection at $t_{s}$ introduces a substantial change in the process due to initiation of foam formation and 
coalescence in the reservoir. Therefore, a fine time grid is required during that time period to capture the sudden changes more accurately. In most of our simulations we took the step-size strategy corresponding to Table A-3 in the Appendix, or a small variation thereof. In our problem, we encountered large intermediate gradients around the times that the spikes (in the injection rate $Q_{g}$ ) occur that have been described in Section 4 (see e.g. Fig. 4.1 or Fig. 4.3). As was illustrated in Fig. 4.2 for the 1D problem, there is a repeating cycle of spikes corresponding to the events that the water saturation $S_{w}$ drops below $S_{w}{ }^{*}$ in successive gridblocks. In Fig. 5.3 we have combined the injection rate profile and a plot of the time gradients $\mathrm{d} J / d t_{k}$ into one plot, clearly showing that the time gradients are (relatively) large at the times that the spikes occur. Apparently, the numerical solution of the (spatially discretized) problem, and in particular the objective function $\mathcal{J}$, is very sensitive to stepsize changes around the times that the spikes occur. As mentioned above, we could reduce the size of these relatively large intermediate gradients by taking even smaller step sizes. As discussed in Section 6 , these spikes do not influence the accuracy of the adjoint gradients at $t_{s}$. In other words, the spikes are an accurate reflection of the behavior of the discretized system.

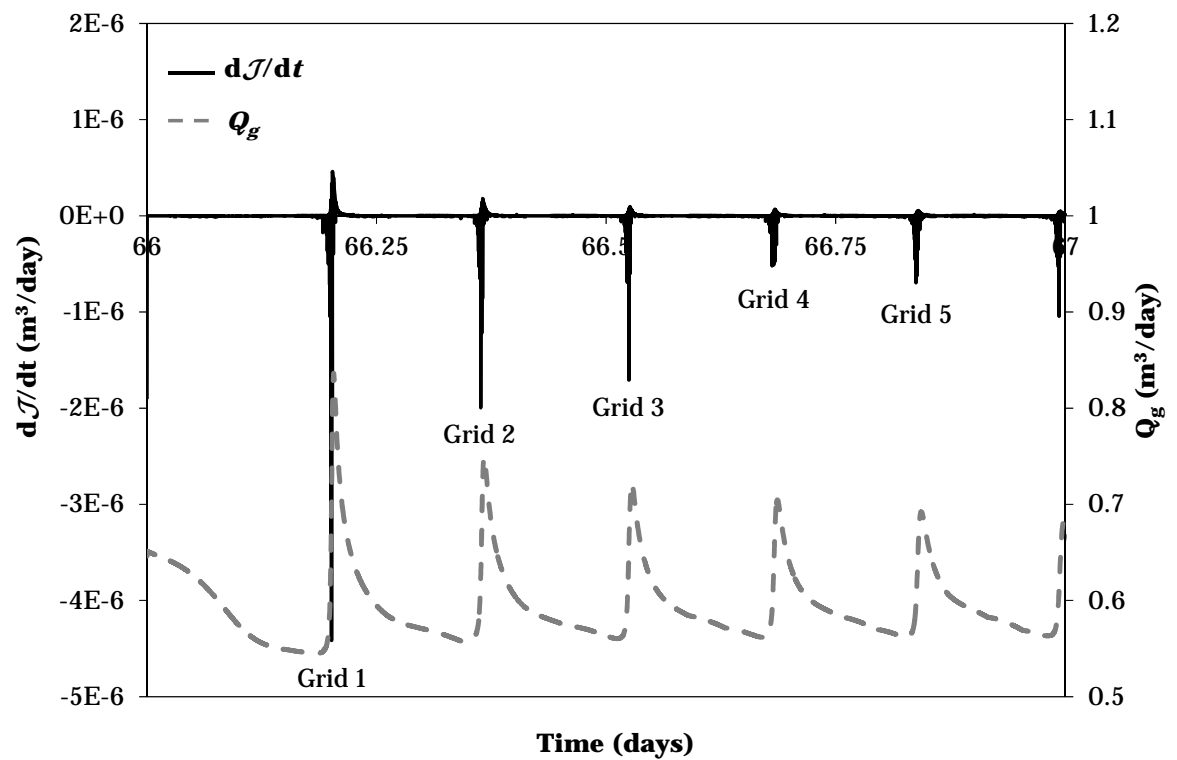

Figure 5.3 Illustration of how fluctuations in gas injection rate correlate with fluctuations in $\mathrm{d} J / d t$ in the $1 D$ simulation (with 100 gridblocks). The moment at which the $n^{\text {th }}$ spike in gas-injection rate occurs corresponds to the moment at which water saturation in the $n^{\text {th }}$ gridblock drops below $S_{w}{ }^{*}=0.316$. Spikes in the time-gradient plot also occur at the same moments. For clarity, we have displayed the gridblock number corresponding to each spike beneath the spike in the time-gradient curve.

\section{Numerical Optimization}

We investigate the capability of a gradient-based, steepest-ascent optimization method applied to our SAG foam process. Our objective is maximizing the cumulative oil production $\mathcal{J}$ by optimizing the switching time $t_{s}$.

We validated the gradients calculated with the adjoint routine in the simulator by comparing them to the numerical gradients obtained with the perturbation method with sufficiently small perturbation sizes, as discussed further below. We use the central-difference scheme for the numerical gradients. We discovered that an inappropriate choice of the relative tolerance for the adjoint linear solver (cf. Section 5.1) is an important source of getting incorrect gradients from the adjoint routine in our problem. We found that $1 \mathrm{E}-12$ is an adequate choice for the relative tolerance for our 1D simulations and for the linear foam model in 3D, but we had to tighten the relative tolerance to its smallest possible value of 2.25E-16 to obtain accurate adjoint gradients for the nonlinear foam model in 3D simulations. Further details are in Namdar Zanganeh (2011). With these relative tolerances we are confident that the adjoint routine computes accurate gradients in our simulations.

During our validation efforts of the adjoint gradients, we encountered a significant number of cases, in which the adjoint gradient did not match the numerical perturbation gradient, suggesting that the 
adjoint gradients were wrong. However, a closer examination (mainly by sampling $\mathcal{J}$ at a finer scale) revealed in all those cases that the adjoint gradients are correct, and that the differences are caused by small-scale local fluctuations in $\mathcal{J}$ superimposed on its global trend. Figure 6.1 illustrates schematically a possible difference between global and local trends of $\mathcal{J}$. By global we mean a trend of $\mathcal{J}$ that is obtained by coarsely sampling of the objective function, by choosing large perturbation sizes. The local trend of $\mathcal{J}$ is obtained by choosing much smaller perturbation sizes than those used for constructing the global trend. In Figure 6.1, the global trend of $\mathcal{J}$ around the two black squares appears to be increasing with increasing $t_{s}$; however, the local trend of $\mathcal{J}$ focusing on the region between the two black squares reveals details that were not noticeable in the global trend.

\section{Global Trend (Coarse Sampling of $\mathcal{J} \quad$ Local Trend (Fine Sampling of $\mathcal{J}$ )}

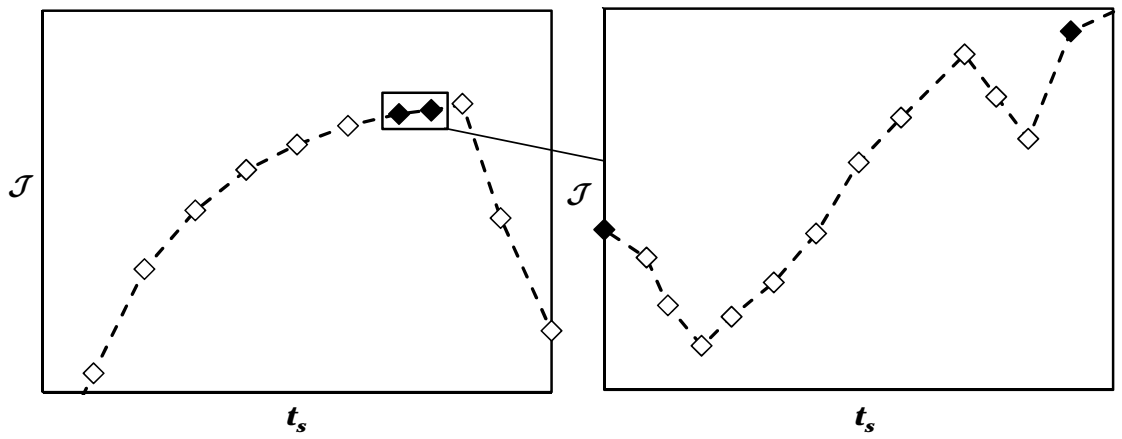

Figure 6.1 Schematic of global and local trends of $\mathcal{J}$ between the two black squares. The local trend of $\mathcal{J}$ differs from the global trend of $\mathcal{J}$ and reveals details that were not noticeable on the global trend.

As we will further discuss below, the observed differences in local and global trend (i.e., the smallscale local fluctuations) of $\mathcal{J}$ will pose a serious challenge for the performance of our adjoint-based steepest-ascent optimization routine for finding the optimum switching time.

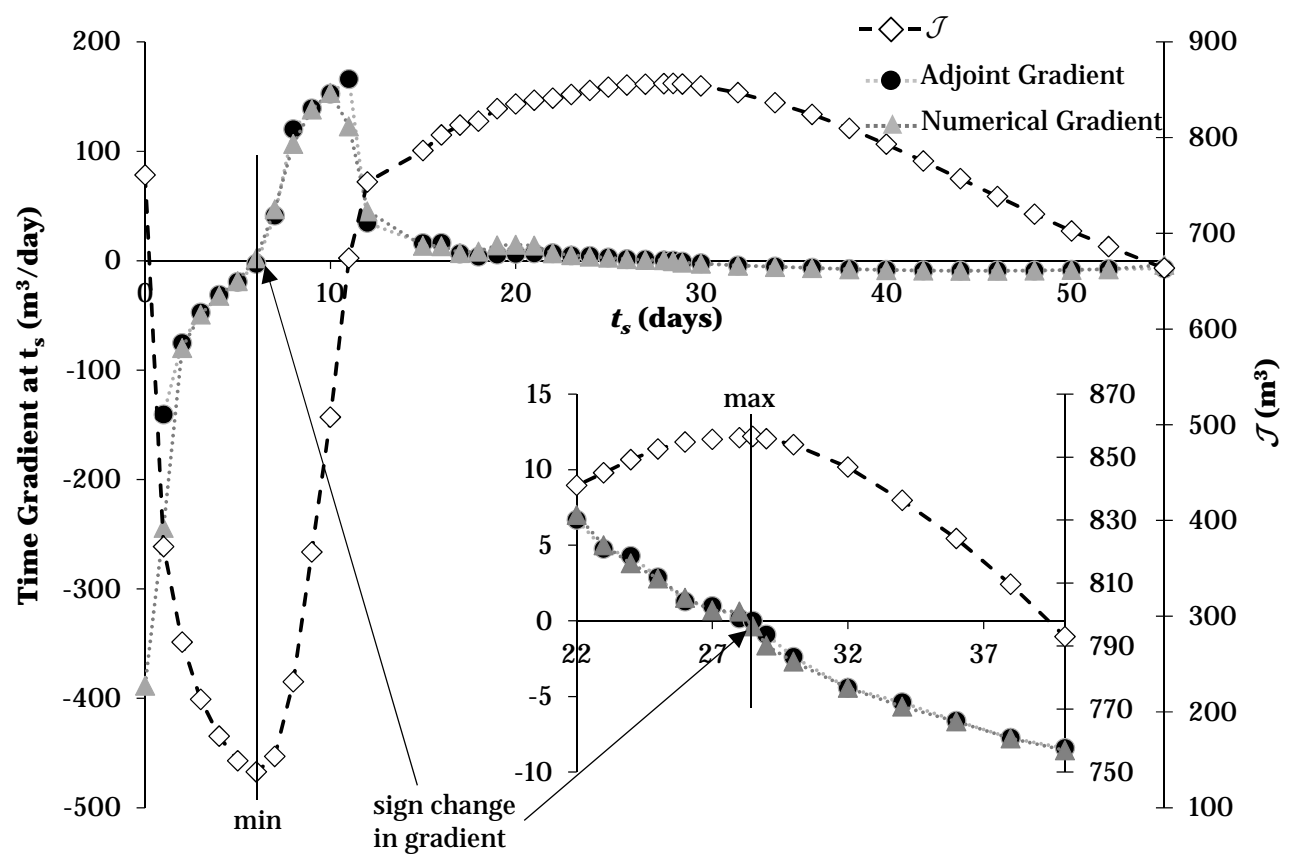

Figure 6.2 Local and global trends of $\mathcal{J}$ for the linear foam model in a $3 D$ reservoir. Total simulation time is 200 days. Except for the initial point at $t_{s}=1$ day, the numerical and analytical (adjoint) gradients are in good agreement. The middle part of the plot around the maximum is magnified in the inset. Both plots share the same axis titles. There is no adjoint gradient at $t_{s}=0$ day, because in that case we start gas injection from the beginning and there is no switching time.

In the subsequent sections we present our findings for the following three cases:

- Linear foam model in a 3D reservoir

- Nonlinear foam model in a 1D reservoir 
- Nonlinear foam model in a 3D reservoir

The case of the linear foam model in a 1D reservoir can be found in Namdar Zanganeh (2011).

\subsection{Optimization with the Linear Foam Model in a 3D Reservoir}

The results for the linear foam model in the 3D reservoir are shown in Figure 6.2. $\mathcal{J}$ has a global minimum at $t_{s}=6$ days and a global maximum at $t_{s}=28.5$ days (magnified in the inset of Figure 6.2). The sign of the adjoint gradients is consistent with the global trend of $\mathcal{J}$. In this case as well as those in Sections 6.2 and 6.3 below, the perturbation size $\Delta t$ for estimating gradients numerically is 1 day before the global optimum and 2 days after the optimum. In Figure 6.2, the adjoint gradient passes through zero at both global optima. Except for the initial point at $t_{s}=1$ day, there is a good agreement between the numerical and analytical (adjoint) gradients. Optimizing the switching time in this process by the steepest-ascent method leads to the correct optimum switching time at 28.5 days.

Similar results are obtained for the linear foam model in a 1D reservoir (Namdar Zanganeh 2011). The steepest-ascent method can successfully find the optimum switching time in both cases.

\subsection{Optimization with the Nonlinear Foam Model in a 1D Reservoir}

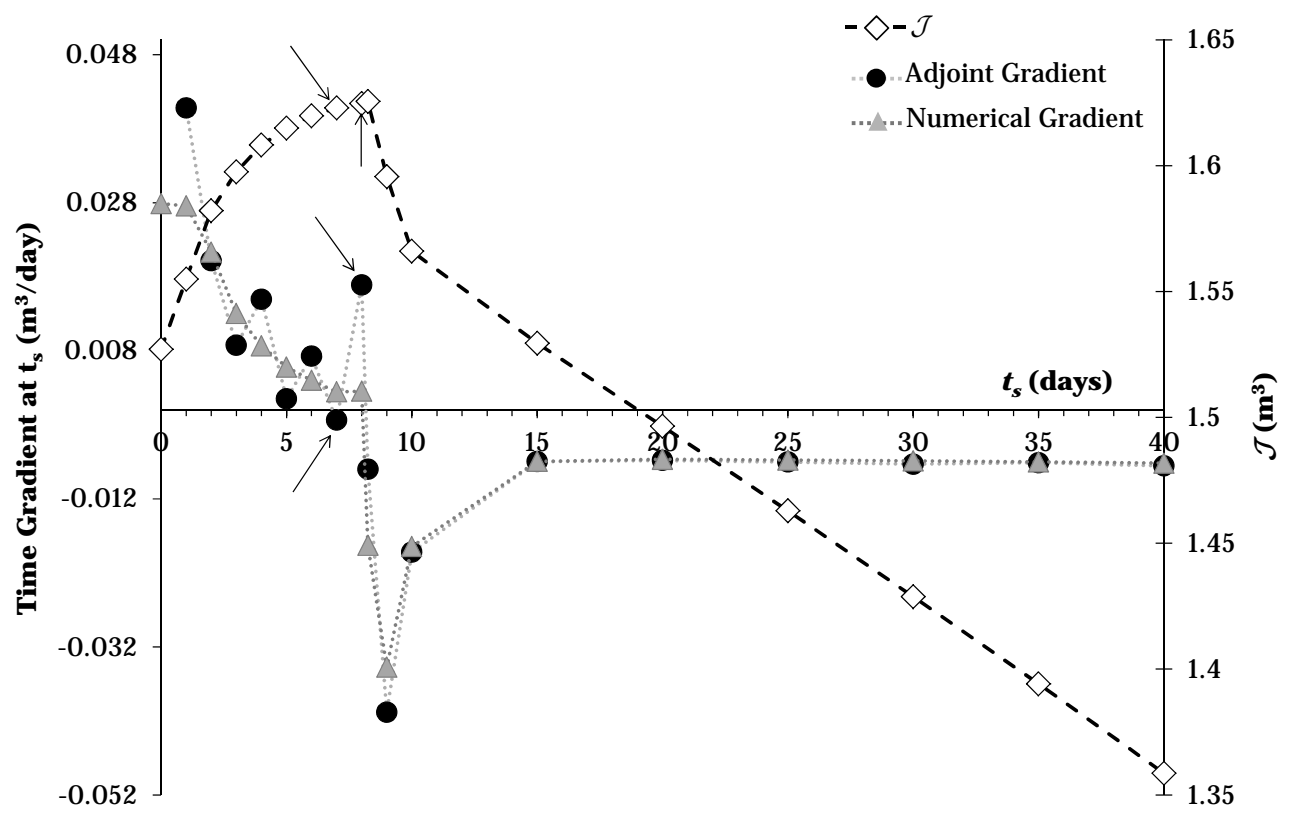

(a)

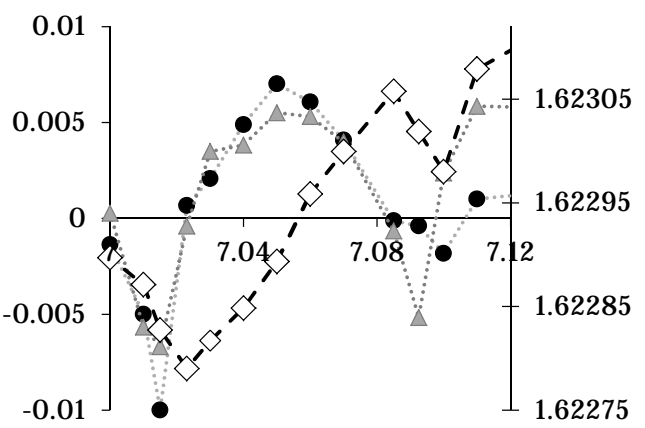

(b)

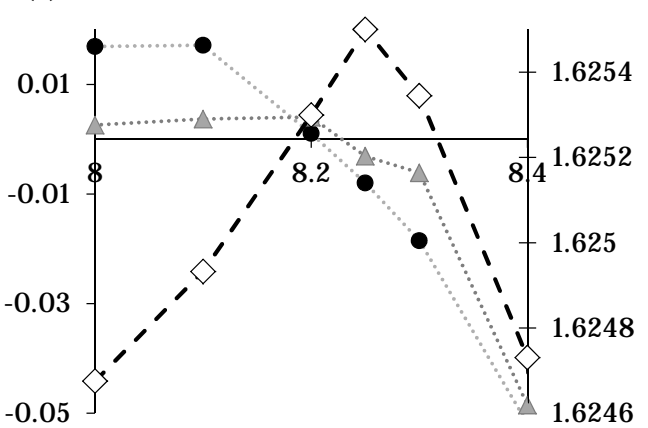

(c)

Figure 6.3 (a) Global trend of $\mathcal{J}$ for the nonlinear foam model in a $1 D$ reservoir. Total simulation time is 200 days. (b) Magnification of the switching time interval from 7 to 7.12 days. A pair of arrows in figure (a) highlights the point $t_{s}=7$ days. (c) Magnification of the region around the global maximum at $t_{s}=8.25$ days. The second pair of arrows in figure (a) highlights the point $t_{s}=8$ days. The adjoint gradient changes sign at this point. Figures (b) and (c) share the same axis titles with Figure (a); we remove them to avoid clutter. There is no adjoint gradient at $t_{s}=0$ day, because in that case we start gas injection from the beginning and there is no switching time. 
The nonlinear foam model produces fluctuations and jumps in the adjoint gradient that are not present with the linear foam model. Figure 6.3 shows the results in 1D. The numerical gradient accurately represents the global trend of $\mathcal{J}$ and it varies smoothly. However, it differs (both in sign and magnitude) from the analytical (adjoint) gradient at certain switching times $t_{s}$. This difference originates from the fact that $\mathcal{J}$ shows fluctuations as $t_{s}$ varies on the scale of 0.01 day superimposed on the larger trend. These fluctuations affect the adjoint gradients and, when taken with sufficiently small perturbations, the numerical gradients.

Figure 6.3a illustrates the global trend of $\mathcal{J}$ in which the global maximum is at $t_{s, o p t}=8.25$ days. There is a negative adjoint gradient and a positive numerical gradient at $t_{s}=7$ days. The global trend of $\mathcal{J}$ appears to be monotonically increasing before $t_{s, o p t}$. However, the expanded view in Figure $6.3 \mathrm{~b}$ reveals that there are two local minima and one local maximum of $\mathcal{J}$ before $t_{s, o p t}$. Also, the magnitude of the numerical and adjoint gradients differ around $t_{s, o p t}$ as shown in Figure $6.3 \mathrm{c}$, because the chosen perturbation sizes are not sufficiently small in this interval.

The global and local trends of $\mathcal{J}$ agree for $t_{s}>10$ days. Therefore, if one approaches the optimum with an initial guess greater than 10 days, the steepest-ascent method can approach the optimum. However, if the initial guess of $t_{s}$ is less than $t_{s, o p t}$, for which the global and local trends of $\mathcal{J}$ do not agree (Figure $6.3 \mathrm{~b}$ ), the correct adjoint gradients may not accurately reflect the global trend of $\mathcal{J}$ that is necessary for finding $t_{s, \text { opt }}$. Therefore, a gradient-based optimization routine might encounter serious problems.

\subsection{Optimization with the Nonlinear Foam Model in a 3D Reservoir}

We now extend the nonlinear foam model to 3D simulations in the presence of gravity. It is helpful to summarize the progress of the flood in 3D. The simulation ends after 540 days. Therefore, the period of gas injection decreases as switching time increases. As in all our examples, injection and production wells operate at fixed bottomhole pressure. For switching times less than 5 days, foam has a marked negative effect because it reduces injectivity without much improvement in sweep efficiency. Foam in these cases sweeps only a region near the injection well. For switching times from 5 to 40 days, foam does improve sweep efficiency, without too much further negative effect on gas injection rate as the foam front advances further from the well. For switching times greater than 40 days, foam reaches the column of gridblocks containing the production well, and after that point in the flood there is a large reduction in gas injection rate as foam reduces the productivity index of the production well. For switching times between 57 and 66 days, foam is able to sweep some oil from the edges of the formation away from the injection well, without much further reduction in injection or production rate. For switching times greater than 66 days, foam is present in the entire formation; increasing the switching time then reduces the period of gas injection with no further harm to injection rate or benefit to sweep efficiency. Details are in Namdar Zanganeh (2011).

The optimal switching time $t_{s, o p t}$ is the switching time for which the foam front stops just short of the production well. For switching times greater than $t_{s, o p t}$, the foam front breaks through to the production well during the process, reducing cumulative oil production below its value at $t_{s, o p t}$.

In the $3 \mathrm{D}$ reservoir with the linear foam model, the gradient-based optimization routine was able to successfully find the optimum switching time. However, Figure 6.4 shows differences between both the sign and the magnitude of the numerical and adjoint gradients at certain switching times. These differences are more pronounced in the 3D reservoir, compared to the 1D reservoir with the nonlinear foam model. We observe a difference in sign of the two gradients near all the optima; i.e., near 5, 40, 57, and 66 days. Having major differences between the global and local trends of $\mathcal{J}$ in the neighborhood of the optimum seriously challenges the optimization routine's performance. These trends agree only after the last local optimum at 66 days. We investigate the local trend for two of these points: the global maximum at $t_{s, o p t}=40$ days and the local maximum at $t_{s}=66$ days, indicated by arrows in Figure 6.4 . 


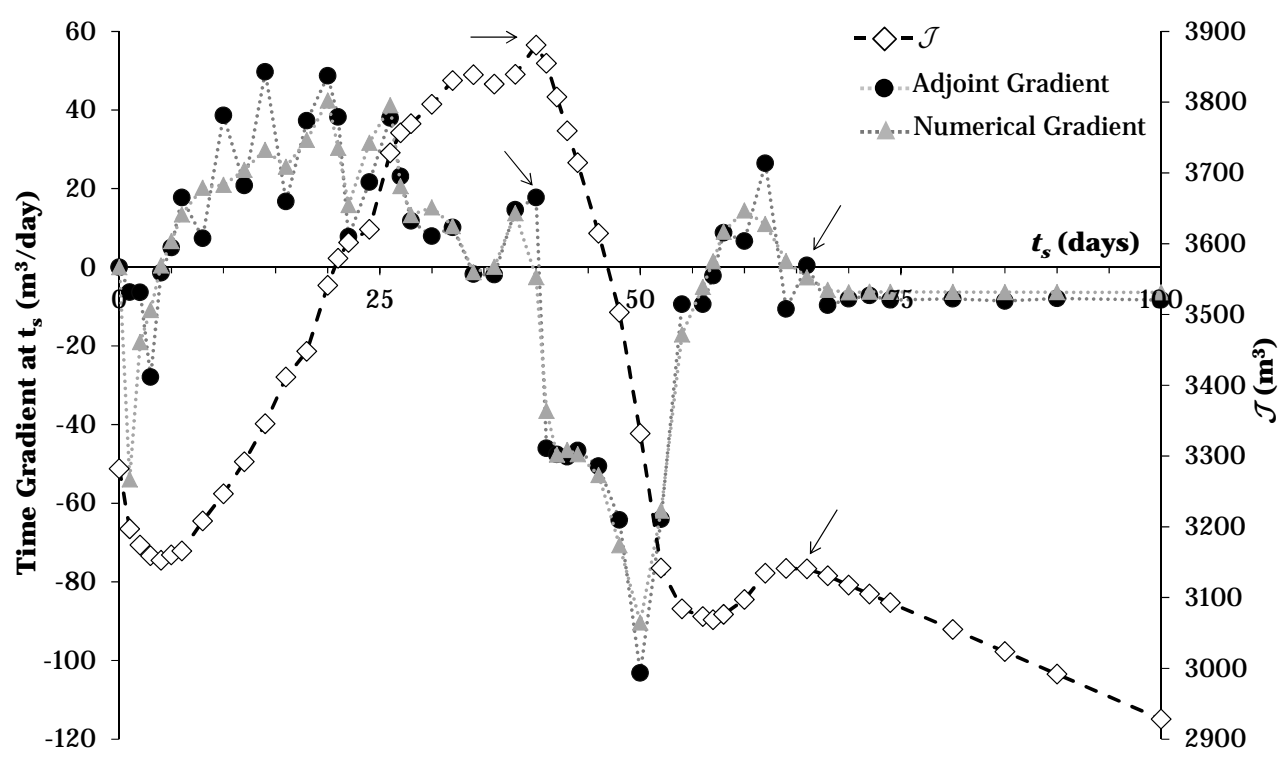

Figure 6.4 Global trend of $\mathcal{J}$ for the nonlinear foam model in a $3 D$ reservoir. Total simulation time is 540 days. The sign of the adjoint and numerical gradients are different near $t_{s}=40$ days and 66 days. The adjoint gradient and $\mathcal{J}$ at these two points are indicated by arrows. There is no adjoint gradient at $t_{s}=0$ day, because in that case we start gas injection from the beginning and there is no switching time.

Figure 6.5 shows the behavior of $\mathcal{J}$ very close to $t_{s}=40$ days. Right after this point, the global trend appears to be decreasing, but the adjoint gradient is positive. However, the local trend around 40 days in Figure 6.5a reveals that $t_{s, o p t}$ is at 40.1 days and not at 40 days. Further, the increasing trend of $\mathcal{J}$ just after $t_{s}=40$ days (Figure 6.5b) is consistent with the positive sign of the adjoint gradient at this point. In addition, Figure 6.5 shows that the numerical gradient is inaccurate at this point; hence, the perturbation size used is not small enough.

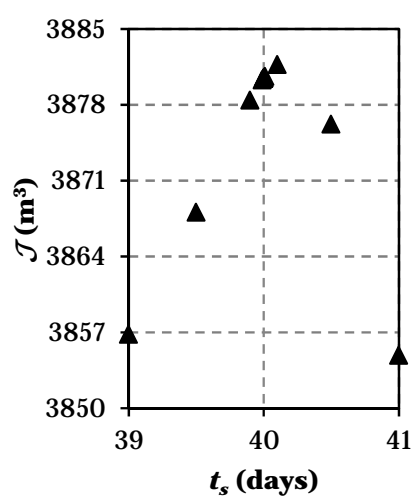

(a)

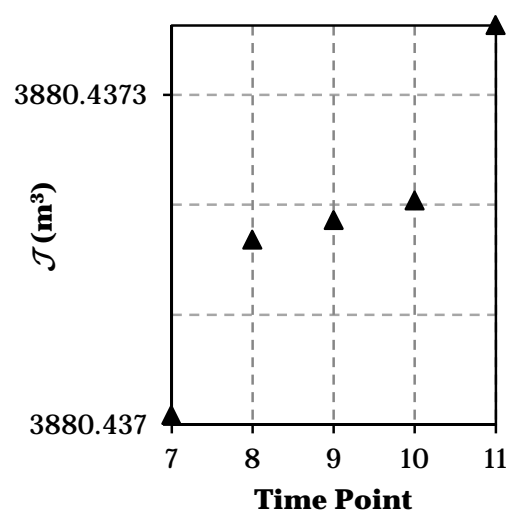

(b)

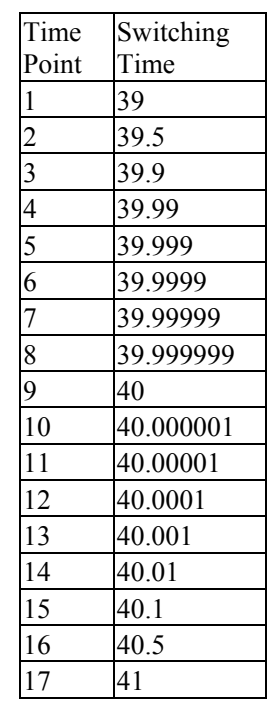

(c)

Figure 6.5 Trend of $\mathcal{J}$ around $t_{s}=40$ days. (a) $\mathcal{J}$ versus the switching time. There are 17 switching times (column 2 of table (c)) in this figure. However, since the middle points are very closely spaced, their trend is not visible in this figure. The correct $t_{s, o p t}$ is at 40.1 days and not at 40 days. (b) $\mathcal{J}$ versus time points identified in column 1 of table(c) in the vicinity of 40 days (i.e., time point 9). (c) Table of switching times and time points used to construct Figures (a) and (b).

Comparing Figures 6.2 and 6.4 reveals that major differences between the global and local trends of the objective function and fluctuations in the adjoint gradient are introduced when the linear foam model is replaced with the nonlinear foam model. We conjecture that in most simulation models, the 
fluctuations on the local trend of $\mathcal{J}$ and the fluctuations in its gradient are small, so that they remain unnoticed. In our case with the nonlinear foam model, where small changes in switching time produce relatively large changes in $\mathcal{J}$, the effects on the gradients are much more pronounced, to an extent that can reverse the sign of the gradient and the adjoint gradients do not reliably lead to a global optimum. That is, the adjoint method gives correct gradients, but these correct gradients may not accurately reflect the global trend of $\mathcal{J}$ that is necessary for finding $t_{s, o p t}$.

Another indication that fluctuations in $\mathcal{J}$ and in gradients derive from the nonlinear foam model is that if foam covers the entire reservoir during the process (i.e., for $t_{s} \geq 10$ days in Figure 6.3 in 1D and for $t_{s} \geq 70$ days in Figure 6.4 in 3D), all the fluctuations in $\mathcal{J}$ disappear and the local and global trends agree. In other words, for surfactant slugs large enough to sweep the entire reservoir: (1) there are no additional, abrupt changes in gas mobility with increasing slug size as foam is able to form in another gridblock, and (2) as it happens, there are no further changes in gridblocks in which foam dries out and collapses with increasing surfactant slug size.

\section{Summary, Conclusions, and Recommendations}

Our objective in this work was to investigate the performance of a gradient-based optimization routine applied to a foam SAG EOR process: specifically, maximizing the cumulative oil production by finding the optimum switching time $\left(t_{s, o p t}\right)$ between the surfactant- and gas-injection cycles, in either $1 \mathrm{D}$ or $3 \mathrm{D}$, with linear or nonlinear foam models. This work is the first attempt to apply optimal control theory to foam EOR processes.

We show that an inappropriate choice of the relative tolerance for the adjoint linear solver is a source of incorrect analytical gradients in our problem, but accurate gradients were obtained with sufficiently small relative tolerance.

We reach the following conclusions from our analysis of the linear and nonlinear foam models:

Linear foam model (3D):

- The local and global trends of $\mathcal{J}$ agree over the entire time interval.

- The chosen perturbation sizes (1 day) for constructing the numerical gradients are sufficiently small for accurate determination of the gradient of $\mathcal{J}$, and they match the adjoint gradients.

- The gradient-based optimization routine is capable of finding the optimum switching time.

Nonlinear foam model (1D and $3 D)$ :

- Replacing the linear foam model with the nonlinear foam model introduced major differences between the local and global trends of $\mathcal{J}$ and fluctuations in the adjoint gradient at some switching times in both $1 \mathrm{D}$ and 3D simulations. The local and global trends agree and the adjoint gradient is free from fluctuations only at switching times for which foam sweeps the entire reservoir within the simulation period. In those cases, the gas mobility-reduction factor makes no further abrupt changes until the end of the simulation once surfactant concentration exceeds the minimum for foam creation in all the reservoir gridblocks.

- In our 1D case, gradient-based optimization is not suitable for finding the optimum switching time, unless the initial guess is larger than $t_{s, o p t}$. In our 3D case, there were major differences between the global and local trends of $\mathcal{J}$ in the neighborhood of the optima that would seriously challenge the optimization-routine's performance. As a result, gradient-based optimization is not suitable for finding the optimum switching time in this case.

- We conjecture that in most simulation models, the fluctuations on the gradient of $\mathcal{J}$ are small, so that they remain unnoticed. In our case with the nonlinear foam model, where small changes in switching time produce relatively large changes in $\mathcal{J}$, the effects on the gradients are much more pronounced, to an extent that can reverse the sign of the gradient and the adjoint gradients do not reliably lead to a global optimum. That is, the adjoint method gives correct gradients, but these correct gradients may not accurately reflect the global trend of $\mathcal{J}$ that is necessary for finding $t_{s, o p t}$.

- The chosen perturbations (i.e., 1 day) for constructing the global trend of $\mathcal{J}$ in the fluctuating zones were not sufficiently small to obtain accurate gradients, except for large switching times, for which the local and global trends of $\mathcal{J}$ were in agreement. Nevertheless, the numerical gradients gave an 
acceptable representation of the global trend of $\mathcal{J}$. As a result, in the cases studied in this work, the numerical gradients would be preferred over the adjoint gradient to be used in the steepest-ascent method for finding the optimum switching time with the nonlinear foam model.

- The appropriate choice of the perturbation size is case-dependent.

- We believe that both the strong oscillations in the forward solution (as described in Section 4) and the small-scale fluctuations in the objective function (as described in Section 6) are caused by the strong variations in gas mobility as described in the nonlinear foam model. It may well be that the encountered problems can be eliminated by the use of a different spatial discretization scheme in the simulator, which is robust against strong variations in gas mobility. However, verifying this and finding a more robust discretization scheme requires further investigation.

The sort of challenges that we encountered with the nonlinear foam model in foam EOR processes could be also present in modeling other EOR processes. Having abrupt changes in properties may cause similar symptoms challenging gradient-based optimization routines in these applications. Obviously, if these symptoms are also observed in those applications, gradient-based optimization routines might not be suitable and other options must be considered for optimizing these processes.

All the complexities and challenges we identified are for a case in which only one control variable is involved. In cases with multiple control variables, a gradient-based optimization routine might face additional challenges.

\section{Acknowledgements}

This research was carried out within the context of the Integrated Systems Approach to Petroleum Production (ISAPP) Knowledge Centre. ISAPP is a joint project between Delft University of Technology (TU Delft), Shell International Exploration and Production (SIEP), and the Dutch Organization for Applied Scientific Research (TNO). We thank Shell Global Solutions International for allowing us to use their in-house reservoir simulator MoReS in this research.

\section{References}

Apaydin, O. and A. R. Kovscek, A.R. [2001] Surfactant Concentration and End Effects on Foam Flow in Homogeneous Porous Media. Transport in Porous Media 43(3), 511-536.

Baker, L.E. [1988] Three-Phase Relative Permeability Correlations. Paper SPE 17369 presented at the SPE Enhanced Oil Recovery Symposium, Tulsa, Oklahoma, 16-21 April.

Bryson, A.E. and Ho, Y-C. [1975] Applied optimal control, Taylor and Francis (Hemisphere), Levittown.

Cheng, L., Reme, A.B., Shan, D., Coombe, D.A. and Rossen, W.R. [2000] Simulating Foam Processes at High and Low Foam Qualities. Paper SPE 59287 presented at the 2000 SPE/DOE Symposium on Improved Oil Recovery, Tulsa, OK, 3-5 April.

Fathi, Z. and Ramirez, W.F. [1984]. Optimal Injection Policies for Enhanced Oil Recovery: Part 2Surfactant Flooding. SPE J. 24 (3): 333-341. SPE-12814-PA.

Fathi, Z. and Ramirez, W.F., [1986] Use of optimal control theory for computing optimal injection policies for enhanced oil recovery. Automatica 22 (1): 33-42.

Fathi, Z. and Ramirez, W. F. [1987] Optimization of an Enhanced Oil Recovery Process with Boundary Controls - A Large-Scale Non-Linear Maximization. Automatica 23(3): 301-310.

Jansen, J.-D. [2011] Adjoint-Based Optimization of Multi-Phase Flow Through Porous Media - A Review. Comput Fluids. 46 (1): 40-51.

Kraaijevanger, J.F.B.M., Egberts, P.J.P., Valstar, J.R. and Buurman, H.W. [2007] Optimal Waterflood Design Using the Adjoint Method. Paper SPE 105764 presented at the SPE Reservoir Simulation Symposium, Houston, Texas, 26-28 February.

Liu, W., Ramirez, W.F. and Qi, Y.F. [1993] Optimal Control of Steamflooding. SPE Adv. Technol. Ser. 1 (2): 73-82. SPE-21619-PA.

Liu, W. and Ramirez, W.F. [1994] Optimal Control of Three-Dimensional Steamflooding Processes. Journal of Petroleum Science and Engineering 11 (2): 137-154. 
Mehos, G.J. and Ramirez W.F. [1989] Use of Optimal Control Theory to Optimize Carbone Dioxide Miscible-Flooding Enhanced Oil Recovery. J. Pet. Sci. Eng. 2 (4): 247-260.

Namdar Zanganeh, M. [2011] Simulation and Optimization of Foam EOR Processes. PhD dissertation, Delft University of Technology, Delft, The Netherlands.

Namdar Zanganeh, M., Kam, S. I., LaForce, T. C. and Rossen, W.R. [2011] The Method of Characteristics Applied to Oil Displacement by Foam. SPE J 16: 8-23. SPE-121580-PA.

Oliver, D.S., Reynolds, A.C. and Liu, N. [2008] Inverse Theory for Petroleum Reservoir Characterization and History Matching. Cambridge: Cambridge University Press.

Ramirez, W.F. [1987] Application of Optimal Control Theory to Enhanced Oil Recovery, Vol. 21. Amsterdam: Development in Petroleum Science, Elsevier.

Ramirez, W.F., Fathi, Z. and Cagnol, J.L. [1984] Optimal Injection Policies for Enhanced Oil Recovery: Part 1-Theory and Computational Strategies. SPE J. 24 (3): 328-332. SPE-11285-PA.

Rossen, W.R. [1996] Foams in Enhanced Oil Recovery. Foams: Theory, Measurement, and Applications, ed. R.K. Prud'Homme and S. Khan. New York: Marcel Dekker.

Rossen, W.R., Zeilinger, S.C., Shi, J.-X. and Lim, M.T. [1999] Simplified Mechanistic Simulation of Foam Processes in Porous Media. SPE J 4 (3), 279-287. SPE- 57678-PA.

Sudaryanto, B. [1998] Optimization of Displacement Efficiency of Oil Recovery in Porous Media Using Optimal Control Theory. PhD dissertation, University of Southern California, LA, California.

Van Doren, J., Douma, S., Wassing, B., Kraaijevanger, H. and de Zwart, B.-R. [2011] Adjoint-ased Optimization of Polymer Flooding. Paper SPE 144024 presented at the SPE Enhanced Oil Recovery Conference, Kuala Lumpur, Malaysia, 19-21 July.

Van Essen, G.M., Jansen, J.-D., Brouwer, D.R., Douma, S.G., Zandvliet, M.J., Rollett, K.I. and Harris, D.P. [2010] Optimization of Smart Wells in the St. Joseph Field. SPE Res Eval \& Eng 13 (4): 588-595. SPE-123563-PA.

\section{Appendix - Model and Simulation Parameters}

Table A-1 summarizes the values for the residual saturations, viscosities, and other parameters in the relative-permeability and foam models. For the two-phase relative permeability of a water/oil system $\left(S_{g}=0\right)$ we have:

$$
\begin{aligned}
& k_{r w}=K_{r w}\left(\frac{S_{w}-S_{w r}}{1-S_{w r}-S_{\text {orw }}}\right)^{n_{w}}, \\
& k_{\text {row }}=K_{\text {row }}\left(\frac{1-S_{w}-S_{\text {orw }}}{1-S_{w r}-S_{\text {orw }}}\right)^{n_{\text {ow }}},
\end{aligned}
$$

and for the two-phase relative permeability of a gas/oil system $\left(S_{w}=S_{w r}\right)$ in the absence of foam, we have:

$$
\begin{aligned}
& k_{r g}=K_{r g}\left(\frac{S_{g}-S_{g r}}{1-S_{w r}-S_{g r}-S_{o r g}}\right)^{n_{g}}, \\
& k_{r o g}=K_{r o g}\left(\frac{1-S_{g}-S_{o r g}-S_{w r}}{1-S_{w r}-S_{g r}-S_{o r g}}\right)^{n_{o g}} .
\end{aligned}
$$

The three-phase relative permeabilities are calculated from the linear isoperm model (Baker 2008). That is, $k_{r w}$ and $k_{r g}$ are defined as above, and oil relative permeability $k_{r o}$ is calculated using linear interpolation of the two-phase oil relative permeabilities $k_{\text {row }}$ and $k_{\text {rog }}$ above.

In the presence of foam, gas mobility is altered from the foam-free value. Foam parameters in Table A-1 are for the nonlinear foam model (Figure 3.1); the simple functions for the linear foam model are easily inferred from Figure 3.1. The nonlinear functions are related to those in the STARS foam simulator (Computer Modeling Group, Calgary, Alberta, Canada) and used by Namdar Zanganeh et al. (2011): 


$$
\lambda_{r g}=\frac{\lambda_{r g}^{0}}{1+f m m o b F_{w} F_{o} F_{s}}
$$

where $\lambda_{r g}^{0}$ is the foam-free relative gas mobility and $F_{w}, F_{o}$ and $F_{s}$ are given by

$$
\begin{aligned}
& F_{w}=\frac{\arctan \left[\text { epdry }\left(S_{w}-f m d r y\right)\right]}{\pi}-\frac{\arctan \left[\text { epdry }\left(S_{w r}-f m d r y\right)\right]}{\pi} \\
& F_{o}=\left\{\begin{array}{cl}
1 & S_{o}<\text { floil } \\
\left(\frac{\text { fmoil }-S_{o}}{\text { fmoil }- \text { floil }}\right)^{\text {epoil }} & \text { floil } \leq S_{o}<\text { fmoil } \\
0 & \text { fmoil } \leq S_{o} \leq\left(1-S_{w r}\right)
\end{array}\right. \\
& F_{s}=\tanh \left(800\left(800 C_{s}\right)^{130}\right)
\end{aligned}
$$

Eq. (A-3) differs from the conventional "dryout" function in STARS (Cheng et al. 2000) in that foam is completely destroyed at $S_{w r}$; for SAG foam processes this is critical (Namdar Zanganeh et al. 2011). At other saturations Eq. (A-3) is similar to the function in STARS. In Namdar Zanganeh et al. (2011), $F_{w}, F_{o}$ are referred to as the "water-killing" and "oil-killing" foam models. Eq. (A-5) gives an abrupt reduction in gas mobility at surfactant concentration $\mathrm{C}_{\mathrm{s}}$ approximately equal to $C_{s}{ }^{*}=0.0012$, which is half the injected surfactant concentration of 0.0024 (weight fraction). Unlike the corresponding function in STARS, it is differentiable at all values of $C_{s}$. It is not an accurate description of foam

\begin{tabular}{|c|c|c|c|c|c|c|}
\hline$S_{w r}$ & $S_{\text {orw }}$ & $S_{o}$ & $\overline{S_{g r}}$ & $\mu_{w}(\mathrm{cp})$ & $\mu_{o}(\mathrm{cp})$ & $\mu_{g}(\mathrm{cp})$ \\
\hline 0.1 & 0.3 & 0 & 0 & $\begin{array}{l}\text { computed } \\
\text { by simulator }\end{array}$ & 5 & 0.02 \\
\hline$K_{r w}$ & & & & $K_{r o g}$ & \multicolumn{2}{|c|}{$K_{r g}$} \\
\hline 0.3 & & & & 0.8 & \multicolumn{2}{|c|}{0.94} \\
\hline$n_{w}$ & & & & $n_{\text {og }}$ & \multirow{2}{*}{\multicolumn{2}{|c|}{$\begin{array}{c}n_{g} \\
3\end{array}$}} \\
\hline 4 & & & & 3 & & \\
\hline fmmob & & & epdry & fmoil $\left(S_{o}^{*}\right)$ & epoil & floil \\
\hline 1,000 & & & 1,000 & 0.4 & 1.5 & 0 \\
\hline
\end{tabular}
strength as a function of surfactant concentration (Apaydin and Kovscek 2001), but it helps to reduce the effects of numerical dispersion on foam simulations (Rossen et al. 1999).

Table A-1 Model parameters. Foam parameters are those for the nonlinear foam model.

Table A-2 gives the reservoir and well parameters for the 3D simulations. The same parameters and properties apply to 1D simulations except the length and number of gridblocks: the 1D reservoir is $100 \mathrm{~m} \times 1 \mathrm{~m} \times 1 \mathrm{~m}$, with $100 \times 1 \times 1$ gridblocks (i.e., $\Delta x=\Delta y=\Delta z=1 \mathrm{~m}$ ), unless otherwise is stated. Prescribed injection-well pressure is 250 bar, and prescribed production-well pressure 145 bar; the initial reservoir pressure is 165 bar. Well injectivity and productivity index are calculated using the Peaceman model.

We applied slightly different time-step sizes for 1D and 3D simulations: to avoid convergence problems, 1D simulations required smaller $\Delta t$ because of smaller gridblocks compared to 3D simulations (Table A-3).

Table A-2 Reservoir and well parameters for the 3D simulations.

\begin{tabular}{|ccccccc|}
\hline $\begin{array}{c}\text { Length }(\mathrm{m}) \\
100\end{array}$ & Width $(\mathrm{m})$ & Height $(\mathrm{m})$ & Depth $(\mathrm{m})$ & $\Delta x(\mathrm{~m})$ & $\Delta y(\mathrm{~m})$ & $\Delta z(\mathrm{~m})$ \\
\hline$\varphi$ & 100 & 30 & 1,600 & 10 & 10 & 3 \\
\hline 0.2 & $k_{x}(\mathrm{mD})$ & & $k_{y}(\mathrm{mD})$ & $k_{z}(\mathrm{mD})$ \\
\hline$P_{\text {ref }}(\mathrm{bar})$ & 100 & & 100 & 10 \\
165 & & $P_{w f}^{\text {inj }}($ bar $)$ & & \multicolumn{2}{c|}{$P_{w f}^{\text {prod }}($ bar $)$} \\
& & 250 & & 145 \\
\hline
\end{tabular}


Table A-3 Time-step strategy for $1 D$ and 3D simulations in Section 6.

\begin{tabular}{|c|c|c|c|c|}
\hline Time Interval & \multicolumn{2}{|c|}{$1 \mathrm{D}$} & \multicolumn{2}{c|}{$3 \mathrm{D}$} \\
\hline & $\Delta t^{\text {start }}($ day $)$ & $\Delta t^{\text {max }}($ day $)$ & $\Delta t^{\text {start }}($ day $)$ & $\Delta t^{\text {max }}($ day $)$ \\
\hline $0 \leq t \leq\left(t_{s}-0.5\right)$ & $1 \mathrm{E}-6$ & $1 \mathrm{E}-2$ & $1 \mathrm{E}-6$ & $1 \mathrm{E}-1$ \\
\hline$\left(t_{s}-0.5\right)<t \leq\left(t_{s}+10\right)$ & $1 \mathrm{E}-4$ & $1 \mathrm{E}-4$ & $5 \mathrm{E}-3$ & $5 \mathrm{E}-3$ \\
\hline$\left(t_{s}+10\right)<t \leq T$ & $5 \mathrm{E}-3$ & $5 \mathrm{E}-2$ & $5 \mathrm{E}-3$ & $1 \mathrm{E}-1$ \\
\hline
\end{tabular}

